\title{
Nuevas perspectivas en la EPOC
}

La enfermedad pulmonar obstructiva crónica (EPOC) es una entidad que puede causar graves efectos en quienes la padecen, y por tanto, en su pronóstico de vida y en sus actividades cotidianas, de ahí que su detección temprana y la instauración de un tratamiento adecuado, mejoren de manera significativa la calidad de vida de estos pacientes.

Con un diagnóstico temprano y el tratamiento adecuado, podría reducirse la mortalidad a causa de la enfermedad pero para esto se requiere avanzar en el conocimiento de la misma y determinar en realidad cuáles son los aspectos fisiopatológicos que deben impactarse para modificar su curso clínico; con modestia expongo una vez más mi punto de vista con relación a que no podemos continuar definiendo el inicio de tratamiento farmacológico únicamente por la presencia o no de síntomas.

El avance más importante en los últimos años es la clasificación del paciente basada en fenotipos, denominación que en época reciente ha tomado cada vez más relevancia para referirse a formas clínicas de los afectados con la EPOC. Esto es motivo de estudio para muchos autores, entre ellos Han en su artículo: "The future of COPD", publicado en el American Journal of Respiratory and Critical Care Medicine, en donde los fenotipos se definen como: «aquellos atributos de la enfermedad que solos o combinados describen las diferencias entre individuos con EPOC en relación a parámetros que tienen significado clínico (síntomas, agudizaciones, respuesta al tratamiento, velocidad de progresión de la enfermedad, o muerte)», visión que probablemente sea la que deba tenerse de esta entidad clínica hoy día. Por tanto, esta clasificación basada en el fenotipo podrá ubicar a los pacientes en subgrupos, lo cual deberá traducirse en valor pronóstico y en una forma más clara de establecer cuál será la terapia más adecuada para obtener una respuesta clínica favorable.

En el reciente enfoque terapéutico también cobra importancia una nueva herramienta conocida como CAT (por la sigla en inglés de COPD Assessment Test), un instrumento de evaluación estandarizado, fundamental en las nuevas propuestas de tratamiento, que puede realizarse de forma rápida y utilizarse en la práctica diaria.

El objetivo que se persigue al aplicar el CAT es medir el impacto de la EPOC en la calidad de vida de los pacientes, permitiéndoles describir sus síntomas con más precisión. La aplicación de este formulario, busca además el acercamiento del médico con el paciente.

La importancia de establecer el fenotipo de un paciente y aplicar el CAT radica en tratar de instaurar un tratamiento dirigido por las características de cada enfermo, lo que se transcribe en un enfoque personalizado del tratamiento farmacológico y de la rehabilitación, aspectos que van más allá de los valores de VEF1.

Con esta nueva alternativa ya no solamente se considera el incremento del tratamiento de acuerdo con la gravedad, sino que además se tienen en cuenta características que hasta hace unos años no se incluían.

Quienes nos enfrentamos a diario a esta enfermedad seguramente coincidimos en que los métodos tradicionales para medir su gravedad son insuficientes y no reflejan adecuadamente sus efectos.

En el pasado, posiblemente al tener en cuenta únicamente el VEF1 como el parámetro más importante para la clasificación, este haya sido la causa por la cual en muchos pacientes no se alcanzaban las metas de tratamiento; con el nuevo enfoque, pese a que aun estamos en deuda con nuestros pacientes, vamos en la dirección correcta, lo cual necesariamente tendría que verse reflejado en un cambio en la evolución natural 
de la enfermedad. Pero para lograr mejores resultados en el futuro se requiere el aporte y, finalmente, el consenso de los especialistas, de modo que se contemplen otras medidas terapéuticas basadas, lógicamente, en las características clínicas del paciente y dirigidas a atacar el evento fisiopatológico más importante de la EPOC, la inflamación.

Robin Rada Escobar, MD. Editor Revista Colombiana de Neumología y Cirugía del Tórax 